\title{
OBSERVATION OF THE ELEMENTAL ABUNDANCES IN LOW-ENERGY COSMIC RAYS IN JULY 1964
}

GPO PRICE \$

CFSTI PRICE(S) S

Hard copy (HC)

Microfiche (MF) f 653 Juty 65

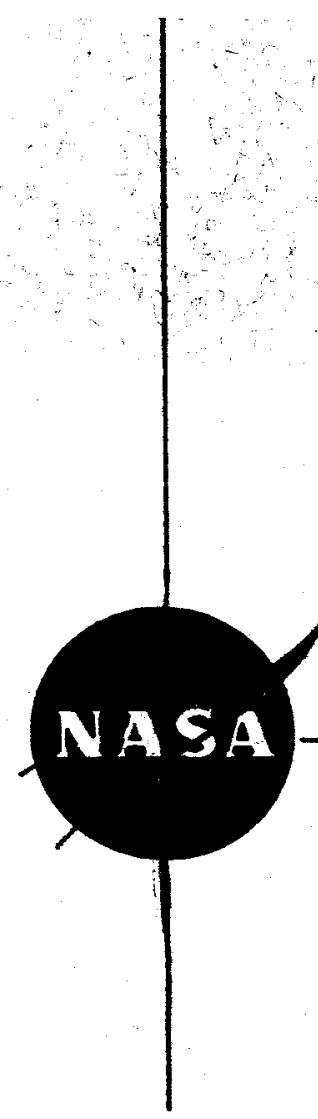

BY

C. E. FICHTEL

D. REAMES
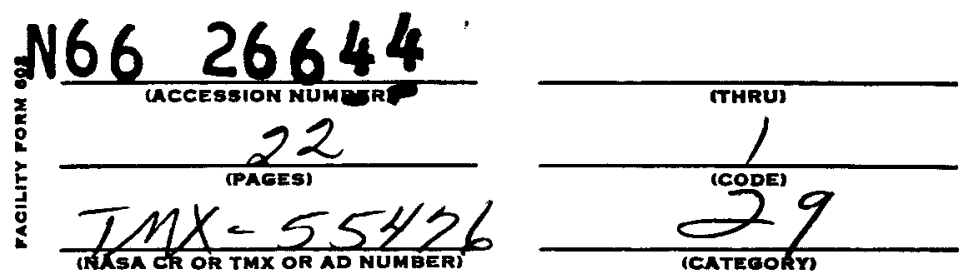

GODDARD SPACE FLIGHT CENTER GREENBELT, MARYLAND 
OBSERVATION ON THE ELEMENTAL ABUNDANCES OF LOW-ENERGY COSMIC RAYS IN

JULY 1964

\author{
D. V. REAMES \\ and \\ C. E. FICHTEL \\ Goddard Space Flight Center \\ Greenbelt, Maryland
}

April 1966 
OBSERVATION ON THE ELEMENTAL ABUNDANCES OF LOW-ENERGY COSMIC RAYS IN JUY 1964

\section{V. Reames and C. E. Fichtel Goddard Space Flight Center \\ Greenbelt, Maryland}

\section{Abstract}

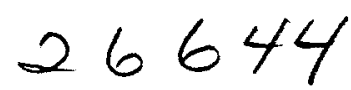

In a sounding rocket experiment flown at Fort Churchill, Manitoba in July, 1964, nuclear emulsion detectors were used to study the composition and fluxes of primary cosmic-ray nuclei with charge $Z \geq 3$ above the atmosphere in the region of kinetic energy from 40 to $220 \mathrm{MeV} /$ nucleon. The results show a light $(3 \leq \mathrm{Z} \leq 5)$ to medium $(6 \leq \mathrm{Z} \leq 9)$ flux ratio of $.16 \pm .07$ in the 40 to $100 \mathrm{MeV} /$ nucleon region and a heavy $(z \geq 10)$ to medium ratio of $.42 \pm .11$ in the 70 to $160 \mathrm{MeV} / \mathrm{nucleon}$ interval. The elemental structure of the medium nuclei is found to contain $(40 \pm 7) \%$ carbon, $\left(10_{-6}^{+4}\right) \%$ nitrogen and $(48 \pm 7) \%$ oxygen. Extrapolating the fluxes back through approximately $2.5 \mathrm{~g} / \mathrm{cm}^{2-}$ of interstellar hydrogen to the source, one finds a flux of nitrogen consistant with zero. 


\section{Introduction}

Much of our present understanding of the origin of cosmic rays and their propagation through interstellar space results from the study of the abundances of multiply-charged nuclei in the cosmic-ray flux at kinetic energies above a few hundred MeV/nucleon ${ }^{1-3}$. It is of particular interest to examine ratios of the fluxes of the different heavy nucle1 (having approximately equal charge to mass ratio) since such flux ratios are insensitive at all energles to the somewhat poorly understood modulation mechanisms which affect the fluxes themselves. The flux ratios in the energy region below $200 \mathrm{MeV} /$ nucleon should be sensitive to the amount of interstellar material traversed, owing to the differing energy loss rates of the particles of different charge, in a way which depends upon the energy spectra of the particles at their source.

The existence of a measurable flux of heavy nuclei in the 20-200 $\mathrm{MeV} /$ nucleon range was demonstrated in a September 1963 sounding rocket experiment flown at Ft. Churchill, Manitoba using large sheets of nuclear emulsion as a detector. ${ }^{4}$ The experiment reported herein was flown in June 1964 using an improved version of the original apparatus to extend the range of measurements to all charges $z \geq 3$ and to unambiguously isolate only those particles of primary origin.

An additional advantage of a sounding rocket observation of cosmic radiation not shared by balloon-boum experiments is that the observed composition is entirely free from secondary particles produced in interactions in the atmosphere. Though corrections for such interactions may be applied to the gross charge groups commonly investigated, the partial cross 
sections for interaction in air are not known on an element by element basis with sufficient precision to determine the detailed elemental composition of the cosmic radiation at the top of the atmosphere. Knowledge of the detailed charge structure of cosmic rays may provide additional information about the source and interstellar propagation of the radiation.

\section{Experimental procedure}

The nuclear emulsion detectors used in this experiment were stacks of $600 \mu \times 6.3 \mathrm{~cm} \times 29 \mathrm{~cm}$ Ilford $\mathrm{G} .5$ pellicles with 18 pellicles per stack. The stacks were arranged in 3 trays which could be extended at a $17.5^{\circ}$ angle with respect to the skin of the Aerobee 150 rocket with 2 stacks per tray. The trays were kept inside the payload until the sounding rocket had left the atmosphere, at which time they were extended. As the trays were extended the top pellicle of each stack was displaced by $1 \mathrm{~cm}$ so that subsequent matching of the tracks would allow the isolation of those which entered during the exposure. The exposure lasted about 350 seconds, after which the trays were retracted and the top plate of each stack was restored to its initial position in preparation for re-entry into the atmosphere. Extension and retraction each took about 7 seconds. The total amount of material above the top emulsion was $.091 \mathrm{~g} / \mathrm{cm}^{2}$ (emulsion equival ent).

After processing, the top plate of each stack was area scanned for the tracks of all particle entering its surface within the dip angle $\left(<65^{\circ}\right)$ and ionization criteria. The minimum ionization accepted was set sufficiently low to insure that all $\mathrm{Z}=3$ tracks would be selected and in practice this meant over half of the alpha particles were also included in the preliminary 
selection. These tracks were then followed into the stack and all tracks which failed to follow (i.e. were displaced by $1 \mathrm{~cm}$ with respect to the top pellicle) or failed to stop in the stack were rejected.

Charges of the particles were determined from delta-ray counting.

If $n_{\delta}(R)$ is the number of delta rays per unit length a distance $R$ from the track ending then a plot of $\log N_{\delta}=\log \int_{0}^{R} n_{\delta}(R) d R$ vs $\log R$ for a given track produces a curve whose shape does not change with the charge (or mass) of the particle if one neglects the small effect of range extension caused by charge pickup. For particles of the same charge to mass ratio one may write

$$
\log \mathrm{N}_{\delta}-\log \mathrm{Z}=\mathrm{F}(\log \mathrm{R}+\log \mathrm{Z})
$$

so that curves for particles of different charge are displaced diagonally from each other by the log of their charge ratio. All charge identifications in this experiment were made using this technique. Delta rays were counted using a parallel-line reticle in the microscope and counting the rays which appeared to extend beyond the lines on either side of a track that was centered on a central line. A count using a narrow line spacing was first made to separate the alpha particles from the higher charges of interest. The narrow line spacing required that the delta rays extend at least $1.8 \mu$ from the center of the track. At least two independent counts were then made for each heavy track using a wider line spacing which selected delta rays extending at least $4.3 \mu$. This spacting was more convenient for counting the heavier tracks. Owing partially to difficulties in counting the high delta-ray densities of the heavier tracks and partially to the effects of range extension mentioned previously, charges of the highly charged particles are underestimated by our method 
of analysis. To correct for these effects a "saturation" correction was applied which increased the measured charges by an amount which varied from a few percent at charge 10 to about $20 \%$ at charge 26 . The results and conclusion of this paper are relatively insensitive to the detailed nature of this kind of correction.

Figure 1 is a comparison of the charges of the particles accepted as determined by two different observers and indicates the charge resolution obtained.

The solid-angle considerations of this experiment are similar to those previously reported ${ }^{4}$ except that all particles with arrival directions making an angle of greater than $90^{\circ}$ with respect to the magnetic field direction were excluded. These particles would be coming from below the rocket and might have resulted from interactions in the atmosphere. The probability of stopping a given particle is a function of its range in the detector, and an efficiency factor, as a function of range, can be compiled from geometrical considerations. This function is essentially flat for ranges from 2 to $12 \mathrm{~mm}$, a lower limit of slightly over $1 \mathrm{~mm}$ being set by the requirement that particles enter the second plate in order to insure their arrival when the rocket was outside the atmosphere. The efficiency function used was found by numerically integrating $\underset{\sim}{\mathrm{n}} . \mathrm{dA} d \Omega$ over the allowed area and solid angle for a given particle range where $n$ is the particle arrival direction. For particles of intermediate ranges the total allowed area-solid-angle factor was $1680 \mathrm{~cm}^{2}$ ster. The upper limit on the energy was determined by the condition that the collection efficiency be greater than $20 \%$ of the maximum value possible i.e. that the range be less than about $2.5 \mathrm{~cm}$. 
Scanning efficiency was determined by a rescan of approximately $25 \%$ of the total area and was found to be between 96 and $99 \%$ for the different observers. Considerable effort was made in the original scan to insure that there be no bias against the light nuclei as evidenced by the large number of $Z=2$ nuclei included in the original sample. No additional light nuclei were located in the rescan and there is no evidence of a charge dependent scanning efficiency. Angular distributions were also searched unsuccesfully for evidence of scanning bias.

\section{Results and Discussion}

A. General Features

Differential fluxes of the light, L, medium, $M$, and heavy, $H$, nuclei collected into $30 \mathrm{MeV} /$ nucleon energy bins are shown in Figure 2 . For comparison with the differential flux values of the $M$ and $H$ nucle1 we also show the smoothed $\alpha$-particle spectra ${ }^{5-8}$ multiplied by the high energy flux ratios, 0.070 and 0.027 respectively. No $\alpha$-particle data taken in July 1964 have been reported for energies below $100 \mathrm{MeV} / \mathrm{nucleon}$ and the curves in this energy region have been obtained by an appropriate averages of data taken before and after this period. 7,8

Ratios of fluxes of various constituent charge groups are shown in Table I together with energy interval from which they are taken. 
Table I

Observed Particle Ratios

Charge Group Ratio Energy Interval

(MeV/nucleon)

$\begin{array}{lcr}L / M & .16 \pm .07 & 40-100 \\ (10 \leq Z \leq 15) / M & .29 \pm .09 & 70-160 \\ (16 \leq Z \leq 19) / M & .02 \pm .02 & 70-160 \\ (Z \geq 20) / M & .11 \pm .05 & 70-160 \\ \text { H } / M & .42 \pm .11 & 70-160\end{array}$

The theoretical implications of these ratios when compared with their higher-energy values are of considerable interest and will be discussed in a subsequent paper.

\section{B. Elemental Structure}

Even a casual observation of Figure 1 reveals considerably finer structure in the charge spectrum than that described by the charge groups discussed above. The elemental structure of the flux of medium nuclei between 40 and $160 \mathrm{MeV} /$ nucleon is shown in Table II along with the "universal" and solar abundances.

\section{Table II}

Composition of the Flux of Medium Nuclei Cosmic-Ray Abundance (this work)

"Universal Abundance" At Source $\left(2.5 \mathrm{~g} / \mathrm{cm}^{2}\right)$ At Earth

$$
40 \pm 7 \%
$$

$\sim 38$

$10+4$

$\sim 0$

$\begin{array}{cc}25.3 \% & 11.2 \% \\ 6.5 & 20.9 \\ 68.1 & 67.9 \\ .004 & .005\end{array}$

$48 \pm 7 \quad \sim 62$
Cameron ${ }^{9}$ $\sim 62$

$\sim 0$

$2 \pm 2$

$\mathbf{F}$ 
It is of interest that data reported from balloon-borne observations of higher-energy cosmic rays indicate a richer nitrogen content than that reported here, while the OGO-I satellite experiments ${ }^{12,13}$ flown more recently confirm our result of $\mathrm{N} /(\mathrm{C}+\mathrm{N}+0) \sim 10 \%$. Even though there are many experiments at high energies whicil glve higher values, some care must be exercised in drawing conclusions about the energy dependence of this ratio since there are several effects that might enhance the observed nitrogen abundance. Not the least consideration is the charge resolution obtained. Many of the balloon experiments were designed only to resolve charge groups and any broadening of the nelghboring carbon and oxygen charge distributions could enhance the apparent number of nitrogen nuclei if not carefully eliminated. Further, the interaction of all nuclei with $\mathrm{Z} \geq 8$ in the residual atmosphere above a balloon would enhance the abundance of $\mathrm{N}$ and $\mathrm{C}$ in a poorly-known way, this effect being aggravated by particles collected during ascent of the balloon in many experiments. Experiments with high charge resolution such as those of Balasubrahmanyan and McDonald 14 and of $\mathrm{O}^{\prime} \mathrm{De} 11$ et al. ${ }^{15}$ each find $\mathrm{N} /(\mathrm{C}+\mathrm{H}+\mathrm{N})=16 \%$, though these experiments may still suffer from effects of the overlaying atmosphere.

Turning attention to the nuclei of even charge one can see from Table II that the $0 / C$ ratio of $1.2 \pm 0.3$ is still considerably smaller than the ratio from universal abundances but is consistent with solar abundances within statistics. Again our data are consistent with the OGO-I results, but apparently inconsistent with the ratios obtained in balloon experiments which typically give $.64^{15}$. As in the case of the nitrogen abundance it remains to be proven that the discrepancy cannot result from 
fragmentation in the overlying atmosphere. One could, in principle, observe the effect of fragmentation in the atmosphere by comparing balloon results from different altitudes but attempts to do so are frustrated by varying degrees of charge resolution and often by the presence of particles collected during ascent.

Above charge 9 we find the composition is characterized by the absence of nuclei of odd charge, with evidence of a higher proportion of $\mathrm{Mg}$ relative to $\mathrm{Ne}$ and $\mathrm{Si}$ when represented in terms of energy per nucleon though both statistics and charge resolution are poorer here than for the medium charge group. In addition to the well-known lack of particles of charges 15 to 19 we also see a gap between the two abundance peaks around charges 20 and 26 . This gap is entirely consistent with universal abundances as are the low fluxes of odd-charged nuclei.

The principal motivation for studying the composition of the cosmic radiation is to learn as much as possible of the elemental composition of the source, thereby to identify it and to study the processes of nucleogenesis in it. There is now considerable evidence that the cosmic rays pass through 2 to $3 \mathrm{~g} / \mathrm{cm}^{2}$ of interstellar material before arriving at earth, and one would like to account for the effects of this material on the composition. Data on the reaction cross sections for protons (interstellar material) incident on various nuclei have been summarized by Badhwar et al.$^{16,17}$ and we have used these data to estimate the abundances of elements, particularly $C, N$, and 0 at the source. The results of these calculations are shown in Table II, the most significant feature being the disappearance of the nitrogen on extrapolating backward 
through about $2.5 \mathrm{~g} / \mathrm{cm}^{2}$ of hydrogen. This effect results primarily from the fact that about half of the inelastic cross section for oxygen-proton reactions leads to nitrogen as a stable product. The relative abundances of the different species of medium nuclei are affected only very slightly by differing energy loss rates and given similar source spectra an energy dependent composition can only be generated by assuming energy dependent cross sections for the production of $\mathrm{C}$ and $\mathrm{N}$ from 0 . Though incomplete, the existing measurements of simflar reactions show no striking energy dependence, or, if anything an energy dependence which might be expected to increase the nitrogen production at low energles. If the fragmentation parameters are assumed to have their high-energy values and if the abundances measured in the low-energy experiments do more correctly reflect the cosmic ray composition outside the atmosphere than do the balioon experiments, then we are left with the conclusions that the source might be almost entirely devoid of nuclei of odd charge with $z \geq 3$ and that the abundance of $\mathrm{N}$, like the abundances of $\mathrm{Li}, \mathrm{Be}$ and $\mathrm{B}$, might be used as a measure of interstellar path length. In stating this result we would caution, however, that the measurement errors are sufficiently large that a source composition with abundances of medium nuclei similar to those given by Cameron cannot be excluded entirely.

\section{Conclusions}

In conclusion, we find that the $L / M$ ratio has attained the low value of $.16 \pm .07$ in the 40 to $100 \mathrm{MeV} /$ nucleon region while the $\mathrm{H} / \mathrm{M}$ ratio has a value similar to that found at higher energies. 
A detailed examination of the cosmic-ray charge structure shows a nitrogen abundance which is not inconsistent with that which would result from fragmentation of the observed $z \geq 8$ nuclei in passing through $\sim 2.5 \mathrm{~g} / \mathrm{cm}^{2}$ of hydrogen. The ratio of the fluxes of oxygen and carbon nuclei is found to be $1.2 \pm 0.3$ in reasonable agreement with satellite measurements in the vicinity of $100 \mathrm{MeV} /$ nucleon but in disagreement with the much lower value found at high energies in balloon experiments. It is not at all clear whether this disagreement results from an energy dependence of unknown origin or from the effect of the residual atmosphere above the balloons. 


\section{Figure Captions}

Figure 1: Scatter plot of particle charges determined by the method described in the test using the measurements of two different observers. Note that all tracks accepted are represented here so that the energy or rigidity interval is a function of charge. Particles with a measured charge less than 2.5 have been suppressed.

F1gure 2: Differential fluxes of light $(3 \leq z \leq 5)$, medium $(6 \leq z \leq 9)$ and heavy $(z \geq 10)$ nuclel in July 1964. Solld curves are the $\alpha$-particle fluxes for the same period (see text) multiplied by 0.070 and 0.027 for comparison with the $M$ and $H$ nulcei respectively. 


\section{References}

1) Webber, W. R., Handbuch der Physik 46.

2) Proceedings of the International Conference on Cosmic Rays, Jaipur, India, Commercial Printing Press, Ltd., Bombay (1963).

3) Proceedings of the Ninth International Conference on Cosmic Rays, London, Sept. 1965.

4) Fichtel, C. E., D. E. Guss, and K. A. Neelakantan, Phys. Rev. 138, B732 (1965).

5) Durgaprasad, N., C. E. Fichtel and D. E. Guss, Proceedings of the Ninth International Conference on Cosmic Rays, London, Sept. 1965, Spec. 19.

6) Waddington, C. J. and P. S. Freier, Proceedings of the Ninth International Conference on Cosmic Rays, London, Sept. 1965.

7) Fan, C. Y., G. Gloeckler \& J. A. Simpson, J. Geophys. Res. 70 , 3515 (1965).

8) Fan, C. Y., G. Gloeck ler \& J. A. Simpson, Proceedings of the Ninth International Conference on Cosmic Rays, London, Sept. 1965.

9) Cameron, A. G. W., Astrophys. J. 129, 676 (1959).

10) Suess, H. E. and H. C. Urey, Revs. Mod. Phys., 28, 53 (1956).

11) Biswas, S. and C. E. Fichtel, Astrophys. J. 139, 941 (1964).

12) Balasubrahmanyan, V. K., D. E. Hagge, G. H. Ludwig and F. B. McDonald, Proceedings of the Ninth International Conference on Cosmic Rays, London, Sept. 1965.

13) Comstock, G. M., C. Y. Fan and J.A. Simpson, Proceedings of the Ninth International Conference on Cosmic Rays, London, Sept. 1965. 
14) Balasubrahmanyan, V. K. and F. B. McDonald, J. Geophys. Res. 69, 3289 (1964).

15) O'Dell, F. W., M. M. Shapiro and B. Stiller, J. Physical Soc. Japan 17, Supp A III, 23 (1962).

16) Badhwar, G. D., R. R. Daniel and B. Vijayakshmi, Progr. Theoret. Phys. 28, 607 (1962).

17) Badhwar, G. D. and R. R. Daniel, Progr. Theoret. Phys. $\underline{30}, 615$ (1963). 


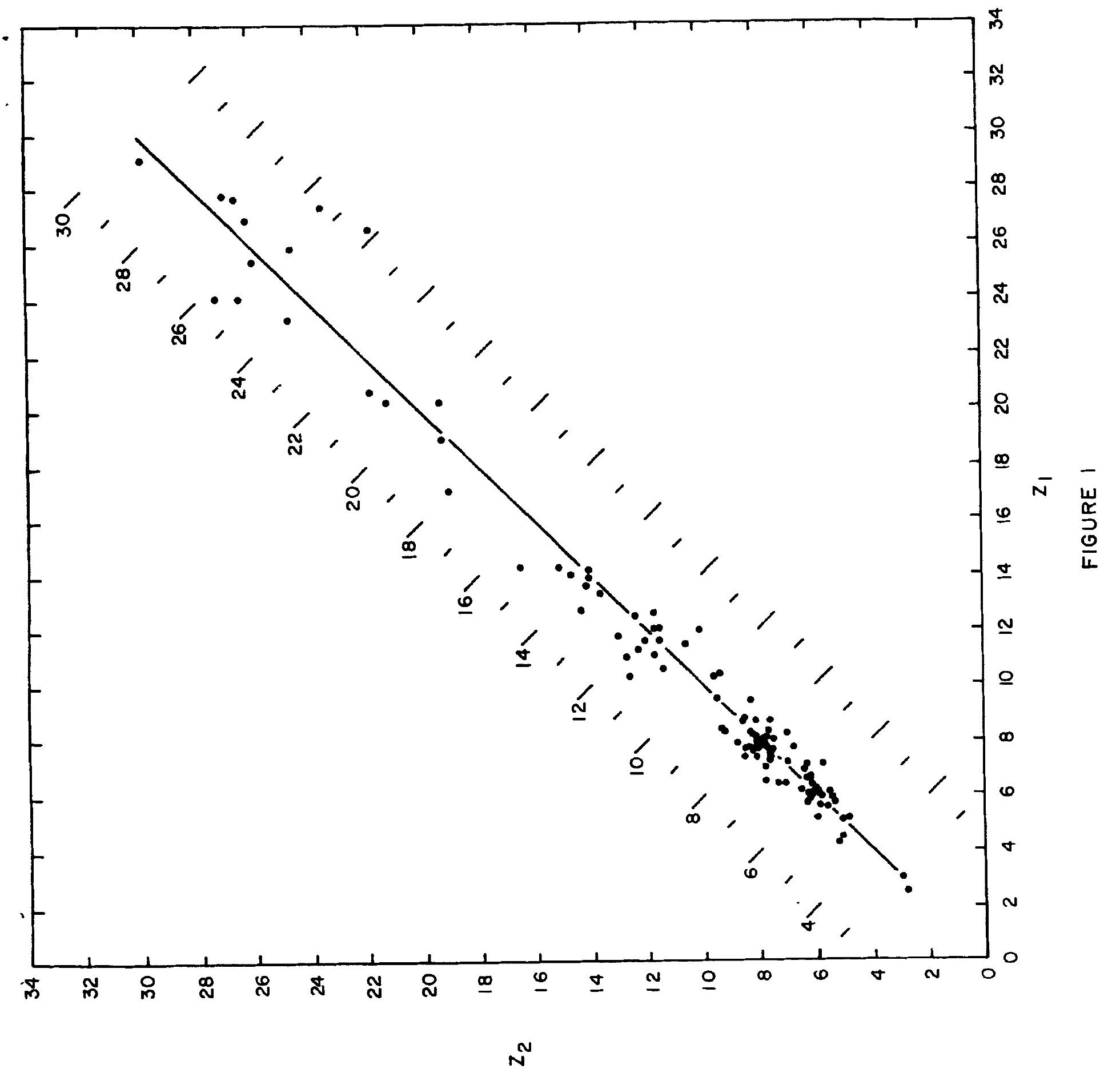




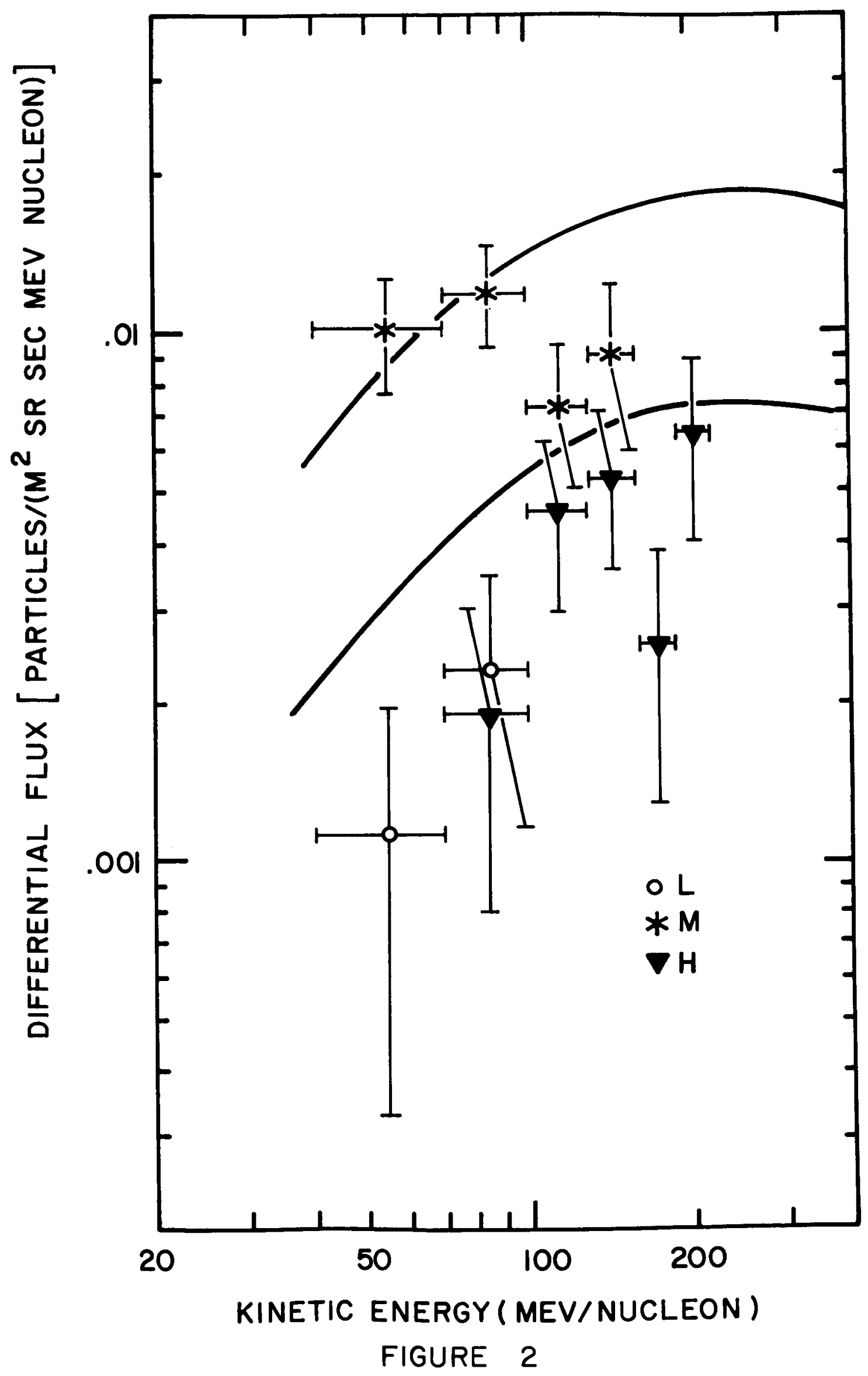

